\title{
Approaches to Address the Operational Limitations of MANETs through Ad Hoc Mobile Cloud Computing Paradigm
}

\author{
https://doi.org/10.3991/ijim.v14i09.14103 \\ K. Muralidhar $(\bowtie)$, K. Madhavi \\ JNTUA, Ananthapuram, India \\ muralidhar.kurni@ieee.org
}

\begin{abstract}
Considering the rising prevalence of mobile devices, Mobile AdHoc Networks (MANETs) are becoming important for wire-less communication. Mobile devices, however, face numerous resource challenges such as bandwidth, mobility, battery life, security and storage. Hence, the service quality enhancement is greatly hindered due to restricted resource availability. MCC makes use of resources in cloud computing systems to address the dearth of native resources in mobile devices. If a connection to the cloud computing network is impassable or high on cost to afford, it restricts a mobile user from incorporating cloud computing services. Therefore, an initial solution will be to use resources from nearby devices instantly. Such a paradigm is known as mobile ad-hoc cloud computing where every mobile device uses its neighboring device's service and resource. This paper shortly explains the contributions done by us to overcome the three vital operational limitations of mobile devices namely connectivity, storage and, processing capability through the Mobile Ad-Hoc Cloud Computing Paradigm. The potential promise in these proposed approaches is evaluated through simulations. Our proposals, taken together intend to increase the operational efficiency of MANETs.
\end{abstract}

Keywords-MANETs, Mobile Ad Hoc Cloud Computing, DaaS, ASaaS, AhCaaS.

\section{Introduction}

Mobile Ad-Hoc Network (MANET) namely a self-configurable network, which consists of wireless hosts where communication facilitated without a centralized administration [1]. However, the inherent nature of MANETs presents many challenges and constraints [2]. The mobile nodes also suffer from various resource constraints. The importance of overcoming the basic constraints of mobile devices/MANETs has long been recognized by the research community. Numerous projects are still in progress, which are working to overcome the constraints of MANETs. However, these projects are getting more and more technically complicated and technically difficult, which exposes construction operations to even a lot of complicated constraints. So, there is a need to make MANETs constraint-free by making the mobile nodes resource-full. 
Mobile Cloud Computing (MCC), is a combination of Mobile Computing and Cloud Computing (CC) and is introduced to make mobile devices resource-full [3];[4]. MCC permits cloud computing resource platforms such as Amazon EC2, Microsoft Azure, and Google AppEngine used to overcome the dearth of native resource restrictions in mobile devices. But with a wireless environment, continuous connection to a remote cloud might not be possible due to mobility and channel characteristics challenges.

The alternate approach is by deploying cloudlet that provides cloud-computing services for both local and remotely connected mobile devices [5];[6] to the cloud. But, in some situations there will be no infrastructure available for cloudlet deployment. Cloud resource provisioning becomes a challenge that needs to addressed at the moment. To address this, we can use an ad-hoc mobile cloud platform such as Mobile Ad-Hoc Cloud (MAC), Mobile Cloud or Mobile Device Cloud (MDC) where no infrastructure is needed and all mobile devices use the services and resources of their neighboring devices. Therefore, this serves as an optimum solution to use the services and resources of nearby mobile devices.

\section{Operational Limitations of MANETs and Proposed Contributions}

As previously mentioned, the intrinsic nature of MANETs has many operational limitations. Of these, few are mentioned below [2]:

- Autonomous and infrastructure-less

- Bandwidth limitations

- Processing capability

- Energy limitations

- Storage

- Mobility

- Connectivity

- Network scalability

- Variable in link capabilities

Such operational limitations must be resolved to make MANET available to the commercial world for implementation. This research considered three vital constraints of MANETs namely connectivity, storage and, processing capability and proposed approaches to overcome the mentioned vital constraints through Mobile Ad-Hoc Cloud Computing Paradigm. Motivated with the flexibility provided by the Ad-Hoc Cloud Computing paradigm, various appropriate models to overcome the limitations of MANETs by making the mobile nodes resource-full are studied in this research. This research studied MANETs and its three vital limitations mentioned above and to overcome these limitations, this research applied the Ad-Hoc Cloud Computing Paradigm to MANETs.

The remnants of this paper is organized accordingly. Section 3 presents an insight on DaaS (Dissemination-as-a-Service) approach, Section 4 presents the ASaaS (Ad Hoc Storage-as-a-Service) and Section 5 presents AhCaaS (Ad-Hoc Computing-as-a- 
Service) approach. Each section presents briefly on the approach, a conceptual demonstration of the proposed approach, algorithm, and, simulations. Section 6 concludes the paper.

\section{Dissemination as a Service (DaaS)}

The first contribution in improving the operational efficiency of MANETs is to address the connectivity problem. In literature, various routing protocols has been proposed for MANETs [7][8]. Routing is only possible whenever the routing protocol identifies a route to the destination in the case where that route exists. High node mobility creates disconnected networks. Routing messages from one part of the disconnected network to another part results in delivery failure. To overcome this issue, an aggressive approach is needed.

A disconnected MANET is called an intermittent connected MANET (ICMANET). This is also known as a delay tolerant network (DTN). Various routing protocols exist for DTNs. They are categorized as follows [9]:

\section{Deterministic}

a) Space-time routing

b) Tree approach

c) Modified shortest path approaches

\section{Stochastic}

a) History or prediction-based approach

b) Model-based

c) Control movement

d) Coding-based approaches

In this work, we preferred history-based forwarding protocols. The probabilistic routing protocol using the history of encounters and transitivity (PRoPHET) [10] is the best-known history-based routing protocols for DTNs. The PRoPHET makes a forward decision by using the delivery predictability set up at every node $\mathrm{M}$ for each destination D. PRoPHET transfers message when delivery predictability value is higher at another node. Even though PRoPHET achieves favourable results it still needs improvements. The main drawback of PRoPHET is, if a node $\mathrm{A}$ is having equal delivery predictability value as that of $\mathrm{B}$, then PRoPHET will forward the message to both nodes A and B, leading to higher resource consumption, the message spread and, duplication. Hence, this problem can be solved by finding an optimal carrier that offers dissemination service to the source so that the replication of messages is avoided. To improve the operational efficiency of MANETs (i.e., dissemination service in a DTN), this work determined an optimal carrier that offers Dissemination-as-a-service (DaaS) to the source node for disseminating messages to the destination [11].

An 'optimal carrier' is defined as "a node that subsequently reaches the destination in the shortest possible time and one that has a high message delivery ratio". To find 
the optimal carrier node two parameters are considered. One is the next probable meet time (Tp) of a node with the destination and the other is the message delivery ratio (MDR) of the node. The Tp value of a node is determined by the previous meet times of that node with destination and MDR is determined by the ratio of the messages it was previously received and delivered.

\subsection{Proposed algorithm}

When a node (say S) encounters two nodes (e.g., A and B) with equal delivery predictability value for a destination (say D) then it initiates the following algorithm to find the optimal carrier. The steps involved in finding an optimal carrier are as follows.

Step 1: S computes the next probable meet time (Tp) of the two nodes by considering the nodes' $\mathrm{k}$ previous meet times with the destination $\mathrm{D}$.

Step 2: If the Tp of the two nodes is equal, then it decreases the $\mathrm{k}$ value by one and re-computes the Tp.

Step 3: Continue this process until the Tp of A and B differ.

Step 4: Next, S computes the MDR of the two nodes,

Step 5: S compares the two nodes Tp and MDR and selects either A or B as the optimal carrier.

\subsection{Simulation and evaluation}

The proposed mechanism is compared with the existing techniques fuzzy PRoPHET [12] and conventional PRoPHET [10]. The comparisons are shown in Figures 1 to 3.

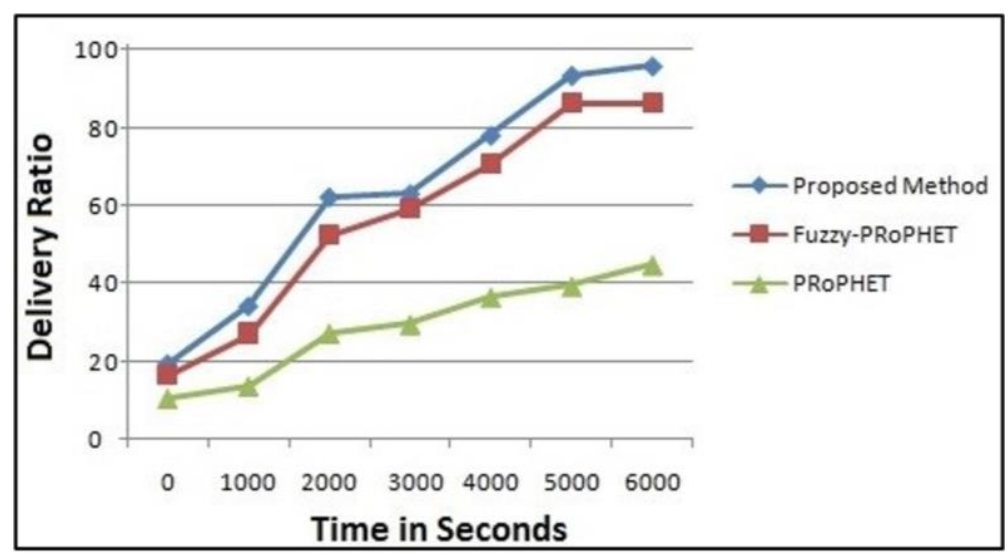

Fig. 1. Packet delivery ratio

The simulation result demonstrates the proposed approach indeed improved the PRoPHET and addressed the connectivity problem with the Dissemination-as-a-Service (DaaS) where an optimal carrier offers dissemination service to the source node 
for disseminating messages to the destination especially in disconnected MANETs (DTNs).

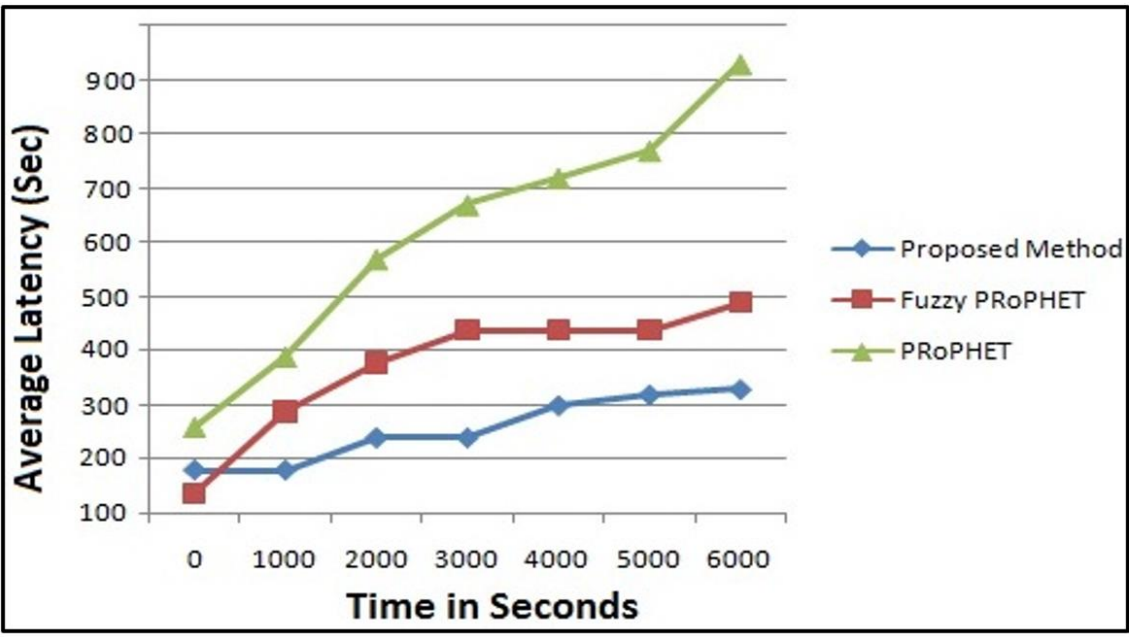

Fig. 2. Average data packet delay

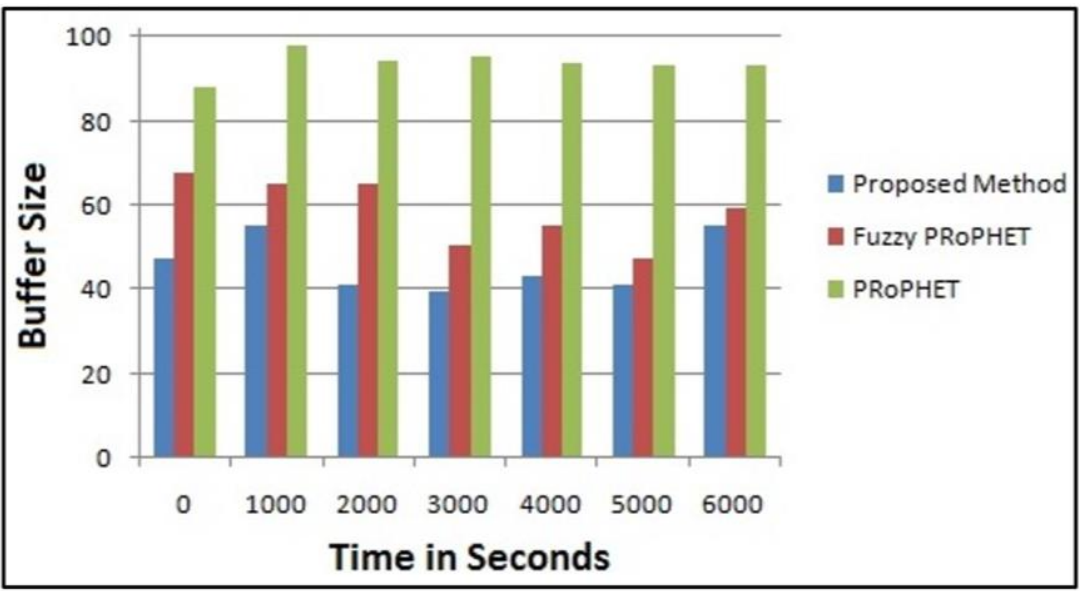

Fig. 3. Average buffer consumption of the nodes in the network

\section{Ad hoc Storage as a Service (ASaaS)}

The Second contribution to improving the operational efficiency of MANETs is to address the storage limitations of mobile nodes in a MANET.

With the growing usage of mobile devices in various domains such as entertainment, education, healthcare and disaster recovery, data growth has increased tremendously. The exponential rise in data is hindered by restrictions in mobile device storage [13]. 
To address this drawback, mobile cloud computing offers infinite storage space by utilizing cloud storage resources. For example, iCloud, Time Machine, Dropbox, etc., facilitate content offloading. However, these services require an uninterrupted internet connection, and may fail in a situation where there is low connectivity and too expensive to afford. Also, continuous internet access in disaster-prone areas and rural areas cannot be guaranteed. The affordable solution in these situations is to use the storage of nearby mobile devices to temporarily store a data backup [14].

Hence for storage and backup system users need to look for an alternate solution in their neighborhood. The proposed ASaaS uses a similar approach to address the storage deficiency problem of mobile devices. In ASaaS [15] mobile nodes with a high volume of storage resources known as Storage Service Providers (SSPs) act as an independent ad-hoc cloud and share their storage to nearby mobile devices, known as Storage Service Requesters (SSRs). SSRs utilize temporary storage/backup services of SSPs. To find a suitable SSP a fuzzy logic technique is proposed. Additionally. the data are encrypted to avoid unauthorized access and stored in the SSPs.

\subsection{Proposed algorithm}

Step 1: Mobile nodes join the network through an 'advertisement' message.

Step 2: When a node (SSR) requires an additional storage/backup it sends a request message to the nodes in the network.

Step 3: The nodes willing to provide their storage replies with a reply message along with information about their residual storage, residual energy, link stability and distance.

Step 4: SSR applies a fuzzy logic approach to select the optimum node (SSP).

Step 5: SSR encrypts data using AES symmetric encryption.

Step 6: SSR forwards the data to SSP through the shortest path.

Step 7: SSP stores the encrypted data of SSR.

\subsection{Simulation and evaluation}

Figures 4 to 9 demonstrate the performance of AsaaS, compared to existing techniques such as SASCloud [16] and Phoenix [17]. 


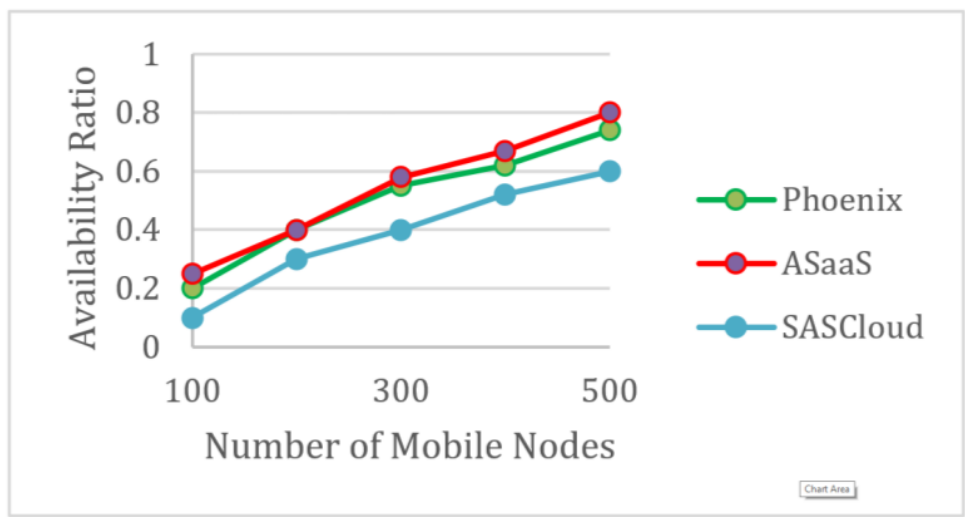

Fig. 4. Availability ratio of data in the network

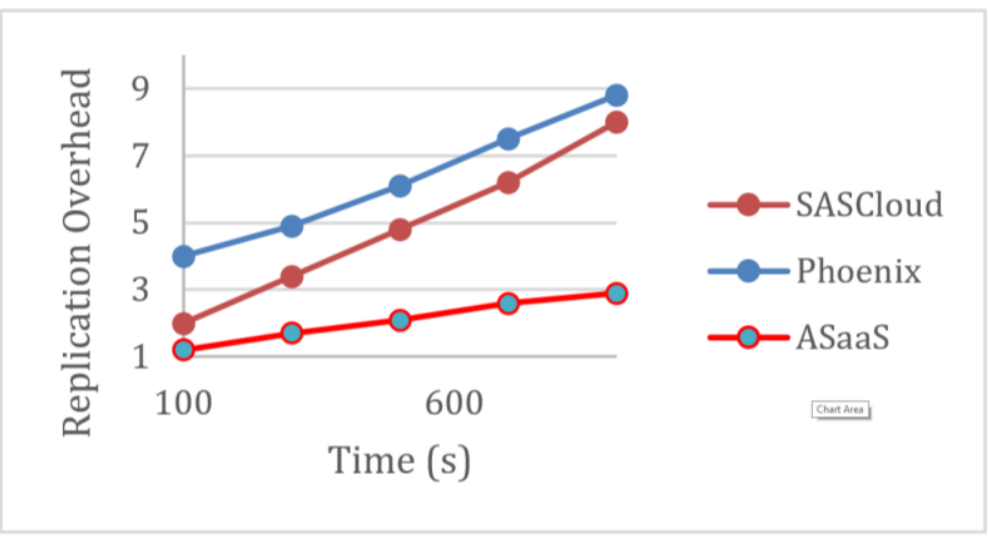

Fig. 5. Replication overhead

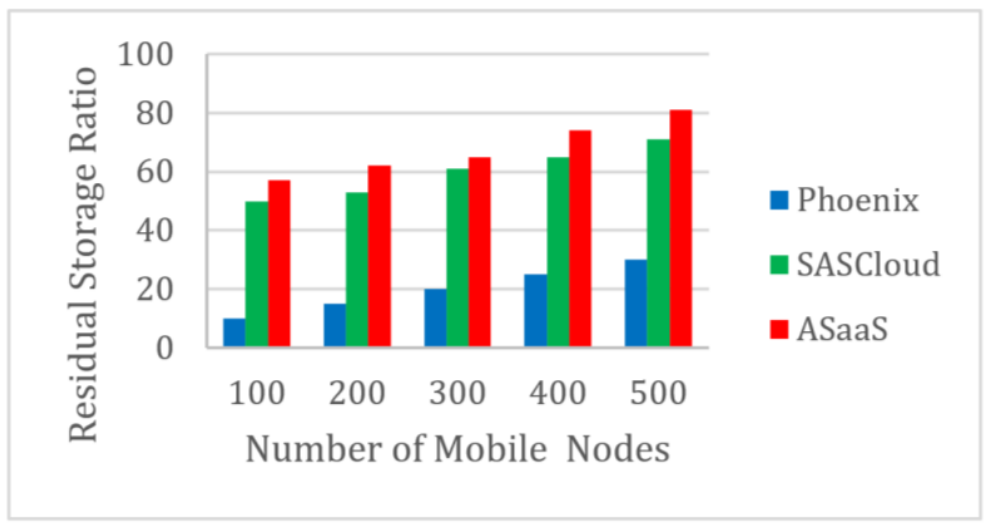

Fig. 6. Residual storage ratio 


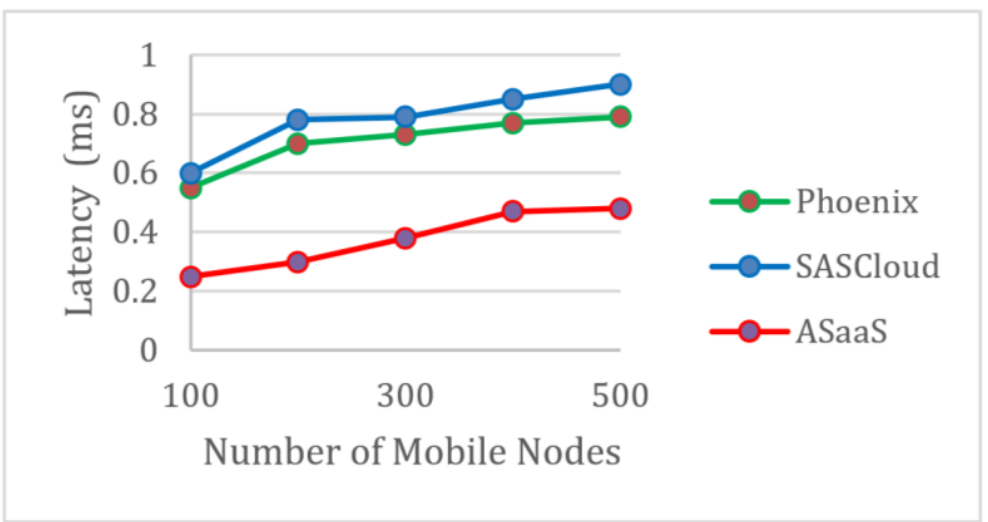

Fig. 7. Latency measurement

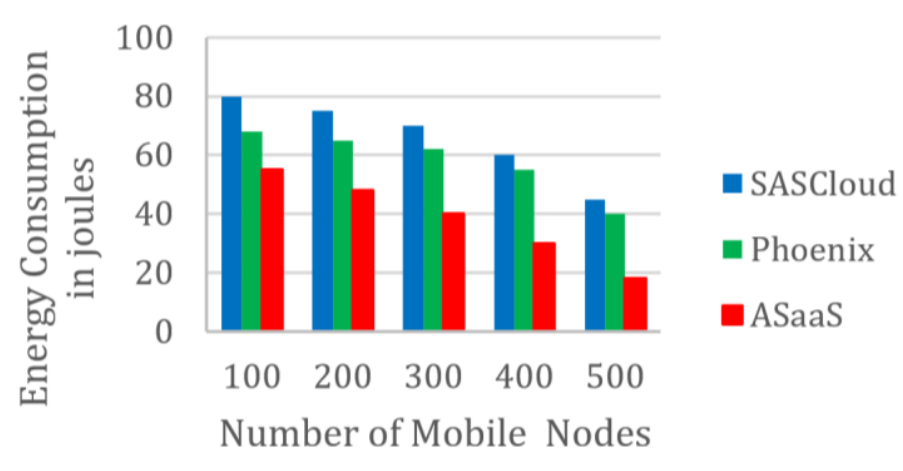

Fig. 8. Overall energy consumption of the nodes in the network

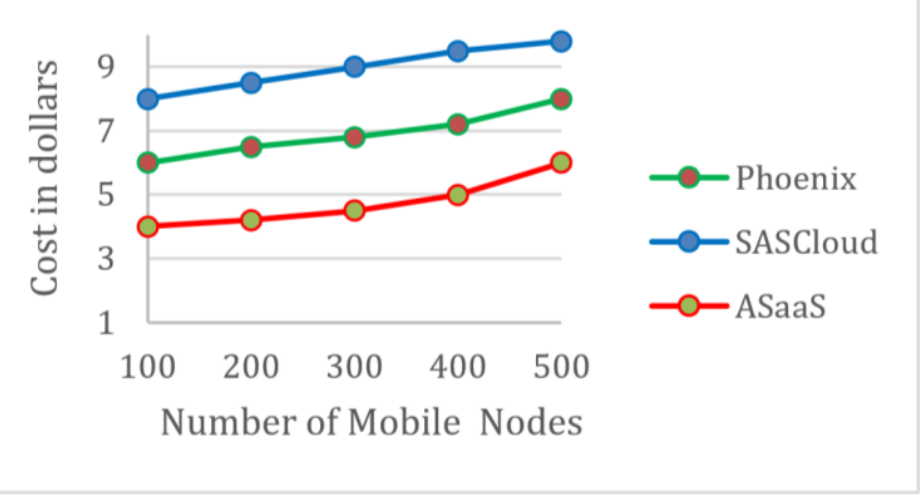

Fig. 9. Cost optimization 
Simulations showed that the proposed ASaaS approach solved the storage deficiency of mobile devices and outperformed the existing mechanism in terms of QoS, energy $\&$ cost.

\section{$5 \quad$ Ad hoc Computing as a Service (AhCaaS)}

The third contribution to improving the operational efficiency of MANETs is to address the deficiencies related to the processing capability of mobile nodes. Since majority of the nodes in MANET are without a superlative processing unit, this problem is addressed through the proposed Ad-Hoc Computing as a Service (AhCaaS) approach [18]. The proposed approach suggests to use nearby mobile devices (mobile phones, PDAs, laptops, etc.) as resource suppliers and take advantage of their capabilities from a different perspective than traditional as a mobile cloud/mobile device cloud. [19] [20].

Through AhCaaS a mobile device with rich processing capability (Offloadee) offers its computation services to other devices with limited resources (Offloader) to process compute-intensive tasks. Offloader offloads the tasks to the nearby by mobile devices that are rich in resources (Offloadee). The main challenge addressed here is selecting an optimal mobile node (Offloadee) for computation offloading. To find a suitable Offloadee a fuzzy logic technique is proposed. The various attributes used for Offlaodee selection are energy efficiency ratio, performance gain, the stability of the node and link stability of the connection.

\subsection{Proposed algorithm}

Step 1: Mobile nodes join the network through an 'advertisement' message.

Step 2: When a node (Computation Offloader) needs to offload computation, it sends a request message to the nodes in the network.

Step 3: The nodes that are willing replies with a reply message along with the information regarding their computation speed, energy coefficient value of the processor (frequency), average processor utilization, and the available duration of the node in the network.

Step 4: Before applying the fuzzy approach, Offloader estimates performance and energy gain.

Step 5: Offloader applies a fuzzy logic approach to select the optimum node (Computation Offloadee).

Step 6: Offloader offloads computation to the Offloadee.

Step 7: Offloadee performs the computation and returns the results to the Offloader.

\subsection{Simulation and evaluation}

The proposed approach is simulated using NS3 and the results are compared with different scenarios and existing approaches. To evaluate the performance of AhCaaS the following evaluation factors are considered: computational time, energy 
consumption, reassignment frequency and, packet delivery ratio. The results are shown in figures 10 to 13 .

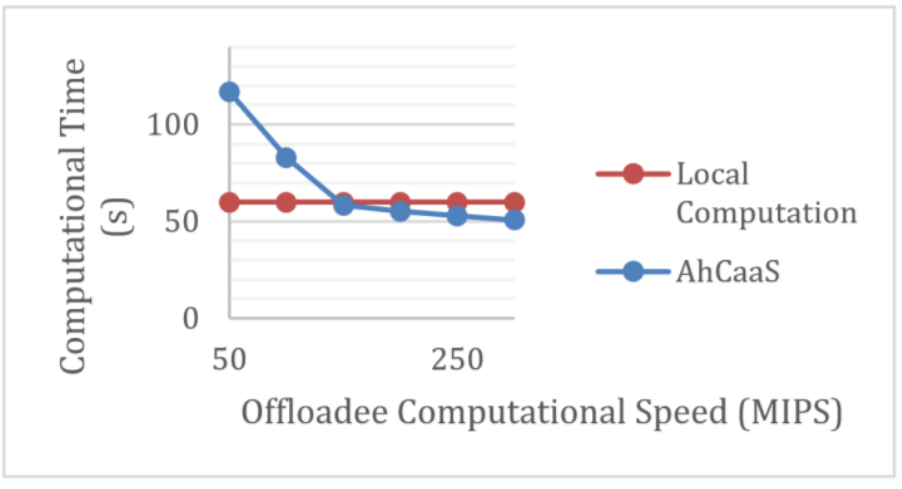

Fig. 10.Impact of increasing offloadee speed on the computational time

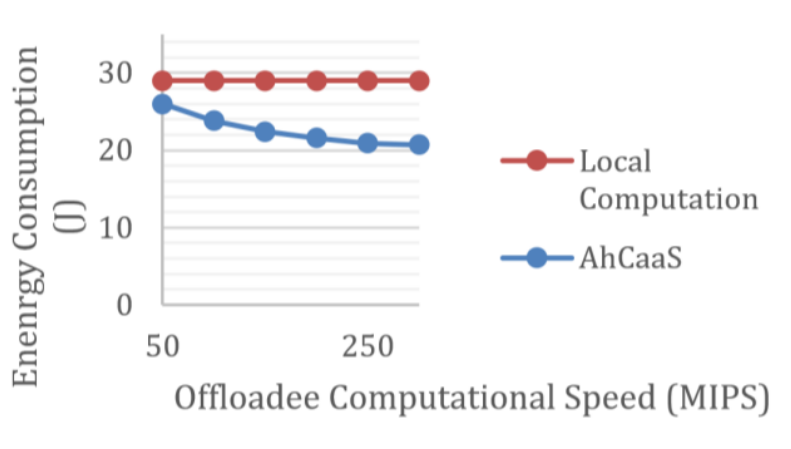

Fig. 11.Impact of increasing offloadee speed on Energy Consumption

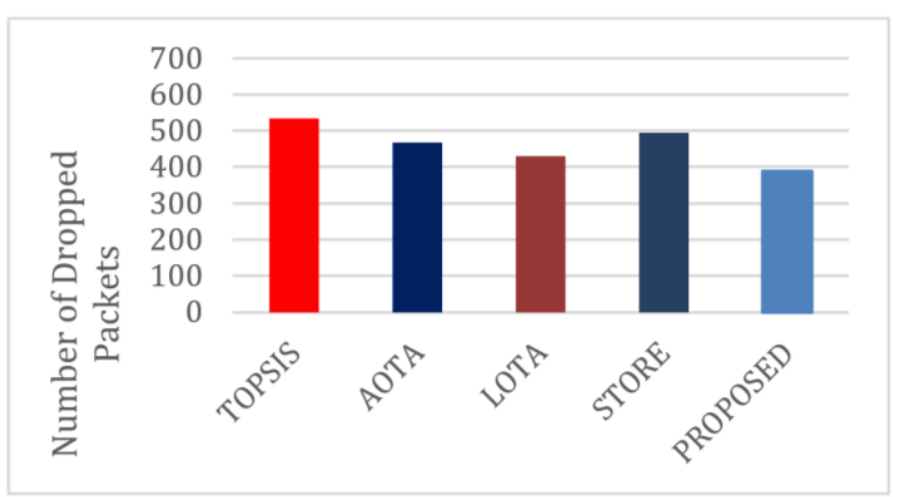

Fig. 12.The total number of dropped packets 


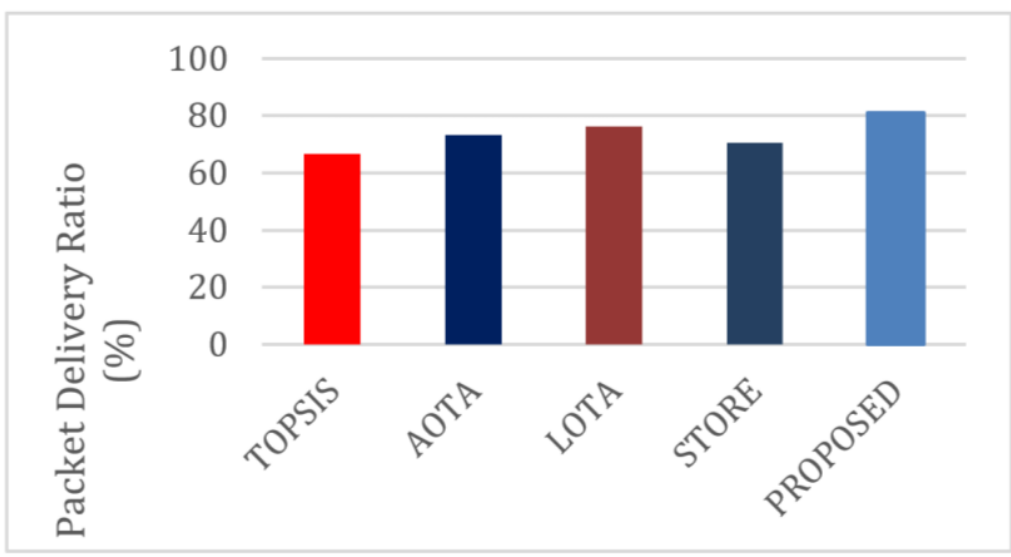

Fig. 13.Packet Delivery Ratio

The results showed that the proposed AhCaaS approach improves the computation speed and also saves energy of the initiating node and also reduces the reassignments with reduced packet drops. The proposed AhCaaS can serve as an alternative for offloading computation to a Cloud or Cloudlet.

\section{Conclusion}

The work presented in this paper is guided by the contributions made by us in improving the operational efficiency of MANETs. We have considered a mobile ad hoc cloud computing paradigm in improving operational efficiency. We presented three approaches in improving operational limitations of mobile devices in MANETs namely connectivity, storage and, processing capability. The work presented in this paper is a step towards improving the operational efficiency of MANETs.

\section{$7 \quad$ References}

[1] K. Muralidhar and N. P. Bharathi, "A relay selection mechanism using RED factors for cooperative communications in mobile ad-hoc networks," 2014 International Conference on Communication and Signal Processing, Melmaruvathur, 2014, pp. 724728.https://doi.org/10.1109/iccsp.2014.6949938

[2] K. Muralidhar and K. Madhavi, "An investigation into the operational limitations of mobile ad hoc networks," 2017 International Conference on Wireless Communications, Signal Processing and Networking (WiSPNET), Chennai, 2017, pp. 13731376.https://doi.org/10.1109/wispnet.2017.8299988

[3] N. Fernando, S. W. Loke, and W. Rahayu, "Mobile cloud computing: A survey," Future Generation Computer Systems, vol. 29, no. 1, 2013, pp. 84-106. https://doi.org/10. 1016/i.future.2012.05.023

[4] EL-SOFANY, Hosam; ABOU EL-SEOUD, Samir. A Novel Model for Securing Mobilebased Systems against DDoS Attacks in Cloud Computing Environment. International 
Journal of Interactive Mobile Technologies (iJIM), [S.1.], v. 13, n. 01, p. pp. 85-98, jan. 2019. https://doi.org/10.3991/ijim.v13i01.9900

[5] T. Soyata, R. Muraleedharan, C. Funai, M. Kwon and W. Heinzelman, "Cloud-Vision: Realtime face recognition using a mobile-cloudlet-cloud acceleration architecture," 2012 IEEE Symposium on Computers and Communications (ISCC), Cappadocia, 2012, pp. 000059000066. https://doi.org/10.1109/iscc.2012.6249269

[6] Y. Jararweh, L. Tawalbeh, F. Ababneh and F. Dosari, "Resource Efficient Mobile Computing Using Cloudlet Infrastructure," 2013 IEEE 9th International Conference on Mobile Adhoc and Sensor Networks, Dalian, 2013, pp. 373-377. https://doi.org/10.1109/ $\underline{\operatorname{msn} .2013 .75}$

[7] KUMAR, Josh; KATHIRVEL, A. Analysis and Ideas for Improved Routing in MANET. International Journal of Interactive Mobile Technologies (iJIM), [S.1.], v. 13, n. 04, p. pp. 164-177, apr. 2019. https://doi.org/10.3991/ijim.v13i04.9928

[8] ABDEL JAWAD, Mada'; SALAH, Saeed; ZAGHAL, Raid. DSDV Extension to Enhance the Performance of Ad Hoc Networks in High Diverse-Velocity Environments. International Journal of Interactive Mobile Technologies (iJIM), [S.1.], v. 14, n. 06, p. pp. 95-114, apr. 2020. https://doi.org/10.3991/ijim.v14i06.11889

[9] Z. Zhang, "Routing in intermittently connected mobile ad hoc networks and delay tolerant networks: overview and challenges," in IEEE Communications Surveys \& Tutorials, vol. 8, no. 1, pp. 24-37, First Quarter 2006. https://doi.org/10.1109/comst.2006. $\underline{323440}$

[10] Lindgren, A., Doria, A. and Schelen, O., "Probabilistic routing in intermittently connected networks," ACM SIGMOBILE Mobile Computing and Communications Review, Vol. 7, No. 3, 2003, pp.19-20. https://doi.org/10.1145/961268.961272

[11] K. Muralidhar, K. Madhavi, "Improving the Dissemination of Messages in MANETs through an Optimal Carrier," International Journal of Networking and Virtual Organisations, Vol. 23, no. 2, 2020; to be published; https://doi.org/10.1504/ijnvo.2020. 10025193

[12] Ahmad, K., Fathima, M., Jain, V. and Fathima, A., "FUZZY-PRoPHET: a novel routing protocol for opportunistic network," International Journal of Information Technology, Vol. 9, 2017, pp.121-127. https://doi.org/10.1007/s41870-017-0021-z

[13] W. Rupert, Mobile Data will Grow 6.3 Times between 2013 and 2018 and Growth will Be Strongest Outside Europe and North America. <http://www.analysysmason.com/AboutUs/News/Insight/Mobile-data-Oct2013/>.

[14] Islam, N. and Aqeel-ur-Rehman, "A comparative study of major service providers for cloud computing," 1st International Conference on Information and Communication Technology Trends (ICICTT), 2013.

[15] Muralidhar, K. and Madhavi, K., "Enhancing the storage of mobile nodes through ad hoc storage as a service in MANETs," International Journal of Communication Systems, 2020; to be published; e4297. https://doi.org/10.1002/dac.42977.

[16] S. Al Noor, M. M. Hossain and R. Hasan, "SASCloud: Ad Hoc Cloud as Secure Storage," 2016 IEEE International Conferences on Big Data and Cloud Computing (BDCloud), Social Computing and Networking (SocialCom), Sustainable Computing and Communications (SustainCom) (BDCloud-SocialCom-SustainCom), Atlanta, GA, 2016, pp. 37-44. https://doi.org/10.1109/bdcloud-socialcom-sustaincom.2016.17

[17] R. K. Panta, R. Jana, F. Cheng, Y. R. Chen and V. A. Vaishampayan, "Phoenix: Storage Using an Autonomous Mobile Infrastructure," in IEEE Transactions on Parallel and Distributed Systems, vol. 24, no. 9, pp. 1863-1873, Sept. 2013. https://doi.org/10.1109/tpds. $\underline{2013.84}$ 
[18] Muralidhar, K. and Madhavi, K., "Setting up Ad hoc Computing as a Service in Mobile Ad Hoc Cloud Computing Environment," International Journal of Interdisciplinary Telecommunications and Networking (IJITN), 2021; to be published.

[19] Bowen Zhou and Rajkumar Buyya, "Augmentation Techniques for Mobile Cloud Computing: A Taxonomy, Survey, and Future Directions," ACM Computing Surveys, Vol. 51, No. 1, 2018, pp. 13:1-13:38. https://doi.org/10.1145/3152397

[20] Ferrer, Ana Juan, Joan Manuel Marquès, and Josep Jorba, "Towards the Decentralised Cloud: Survey on Approaches and Challenges for Mobile, Ad hoc, and Edge Computing," ACM Computing Surveys (CSUR), vol. 51, no. 6, 2019, 111. https://doi.org/10.1145/ $\underline{3243929}$

\section{Authors}

K. Muralidhar is a Research Scholar, currently pursuing his Ph. D from JNTUA, Ananthapuram, India. He received his Master in Computer Science from JNTUACEA, JNTUA. His research interests include mobile ad hoc networks, wireless sensor networks and cloud computing. Email: muralidhar.kurni@ieee.org

K. Madhavi is an Associate Professor at Department of CSE, JNTUACEA, JNTUA, Ananthapuram, India. She received her Ph. D in CSE from JNTUA. She has more than 16 years of teaching experience in computer science and engineering. Her work has been published in several peer reviewed journals and conferences. Her research interests include mobile ad hoc networking, computer organization, big data and cloud computing.

Article submitted 2020-03-06. Resubmitted 2020-04-21. Final acceptance 2020-04-21. Final version published as submitted by the authors. 